\title{
Network structure, capacity growth and control: the case of China's air traffic system revisited
}

\author{
Hans Huber \\ CESUR, \\ Instituto Superior Técnico, \\ Av. Rovisco Pais, 1049-001 Lisboa, Portugal \\ E-mail: hhuber@civil.ist.utl.pt
}

\begin{abstract}
Recent applications of Cournot models suggest that fully connected networks are less costly and more welfare-efficient as compared to hub-and-spokes in China's rapidly expanding air traffic system (ATS). The author applies agent-based heuristics to demonstrate that the complexity of China's ATS can: 1) be decomposed into meaningful components; 2) be graphically depicted as such; 3) not only determine whether HS are in fact dominant or not, but also identify intermediary or more developed forms of HS; 4) make both plausible and coherent inferences about the overall strategy of China's aviation policy. One salient theme that underlies such heuristics is that of path dependency and evolutionary development. Theories related to market liberalisation, entry/exit to the industry or competition versus cooperation including their spatial dimension - can thus be put into perspective within the complex context of Chinese ATS.
\end{abstract}

Keywords: Chinese air traffic; hub-and-spoke; network decomposition; high frequency development; spatial growth.

Reference to this paper should be made as follows: Huber, H. (2013) 'Network structure, capacity growth and control: the case of China's air traffic system revisited', Int. J. Management and Network Economics, Vol. 3, No. 1, pp.39-56.

Biographical notes: Hans Huber is an Associate Professor at SJMSOM (Indian Institute of Technology - Bombay) until recently and currently a Senior Marie-Curie Research Fellow at CESUR (Instituto Superior Técnico - Lisboa). His interests relate mostly to network strategy and industry structure in the context of aviation. His research amounts to over 40 papers and case studies. He holds a PhD from HEC, University of Geneva, Switzerland and an MBA from UC Berkeley.

\section{The Cournot approach and HS}

Zhang (2010) compares fully-connected (FC) with hub-and-spoke (HS) networks for China's domestic air traffic. One main result states that, although HS networks may provide some competitive advantages, it would take a larger investment into infrastructure to operate HS as compared to FC networks [Zhang, (2010), p.196]. Before 
taking issue with this significant conclusion, one has to address the underlying model through which this result is obtained, i.e., the Cournot model of competition.

\subsection{The Cournot model in air traffic}

The author refers to the commonality of the Cournot model when conducting scholarly research into airline competition at specific airports (footnote 2, p.191). Airlines would typically compete in city-pair markets, with carriers setting flight schedules before determining prices to fill the planes in a second stage (p.192). 'Full price' (including ticket price, costs for schedule and congestion delays) is driven both by flight frequency and a constraining element in the form of a volume/capacity ratio at both origin and destination airports (p.191f). Airlines' flight frequencies to/from given airports are thus considered central for optimising the model. Simplifications with regards to real scheduling and pricing are made, i.e., assuming symmetric city-pair traffic or carriers being equal with a constant number of seats and full or constant load factors. One may find issue with one or several of such simplifications, but in the interest of the model application to remain 'readable' this may be acceptable. Cost functions, which determine the airlines' profit, are composed of the individual airlines' operating cost per flight and the airport charge per aircraft at the origin and destination airports (p.192).

The model then is being applied separately to both network types (FC versus HS), and is being solved largely by differentiating w.r.t. frequency and finding the first-order condition for optimum airline output (choice of schedule) in relation to constrained capacity at the respective airports. In the case of an HS configuration, all traffic is being routed through a single hub airport with the assumption that all 'symmetric' carriers would share the same hub city (p.193). As spoke-to-spoke passengers would need to pass through the hub airport, total frequency within the network would increase by definition. Another simplification assumes usage of a single type of aircraft throughout the entire HS system (p.193). Solving for optimal frequency at spoke airports within the HS network arrives at the same result as with FC, i.e., optimal capacity would need to be such that the marginal reduction in congestion costs is equal to marginal costs of capacity for the respective airport investments.

\subsection{Optimal capacity at hub airports}

To derive optimal capacity at the hub airport, direct effects of airport charge and capacity investment on social welfare need to be calculated. With spoke airports, these direct effects net out to equal zero, as they are "in fact an internal transfer between the passengers and the airport authority" (Zhang, p.195). This situation is different at hub airports due to a share of connecting passengers who pay airport charges without obtaining (local) benefits (p.195) - in contrast to the utility passengers obtain when reaching their final tourism/business destination. In that sense, the direct effect of airport charge on social welfare for the hub airport is reckoned to become marginal revenue contributed by the connecting passenger to the hub airport (p.195).

Indirect effects of airport charge and capacity investments are reflected in carriers' output reactions, with the optimality condition for hubs being different from those of spoke airports. Zhang arrives at the conclusion that capacity at hub airports is larger than socially efficient in the sense that the marginal reduction in congestion costs is lower than the marginal cost of capacity (p.195). 


\subsection{Improving social welfare in congested HS networks}

Through the Cournot approach, a transformational logic can be inferred, although Zhang stops short ${ }^{1}$ from doing so: in the case of (congested) HS, social welfare would be improved by refraining from very costly expansion and by re-allocating such traffic on to direct spoke-to-spoke. Such measures would incrementally move the network away from a HS towards a more FC topology instead.

Wei and Hansen (2006, p.842) had highlighted the potential benefits of selectively de-peaking traffic at hub airports to reduce airport congestion and air travel delays. They wrote: “...capacity expansion projects at hub airports are always a priority in government funding allocations. While billions of dollars have been invested in airport capacity expansion projects, surprisingly, there are very few studies in quantifying the benefit of these projects". Different from the analytic model shown by Wei and Hansen, Huber (2009, p.390) seeks to structurally de-emphasise the type of traffic that was a key feature of HS networks, i.e., connecting traffic. As with Cournot, Huber (2009) reckons the role of ticket prices to be secondary to the actual scheduling of capacity on certain routes. Going beyond Cournot, Huber (2009) believes geo-spatial factors, supply-side economics and demographics, i.e., potential demand, to be more important w.r.t. emerging economies.

In particular, Huber $(2009$, p.390) makes a case for Statistical Mechanics to provide amore adequate reference when highlighting misallocation of airlines' capacity (frequency) that may lead to excessive traffic concentration at hub airports. Moreover, statistical mechanics also provides guidance for showing the impact of possible re-allocation of such investments, for example when switching strategies from costly hub expansion to upgrading and modernisation of less congested airports where marginal welfare benefits could be expected to be greater.

\section{A network and agent-based perspective}

The applied Cournot model used differential calculus as the central approach for mathematically 'solving' a simplified representation of extremes of alternative network structures and optimising them w.r.t. social welfare. This paper seeks to include more characteristics of the Chinese ATS to represent it more realistically, mainly by focusing on its distinct topologies and by relating them to its constituent agents. The paper does not intend to present a definite model of domestic air traffic in China, but goes to great lengths in decomposing it and analysing its agents at separate points in time. The fact that these different entities (agents) interact, not only with each other, but also with their local and connected environment is a fundamental feature of complex systems [Railsback and Grimm, (2011), p.10]. New insight is to be gained as far as behaviour of the distinct agents is concerned and how the complex system may adjust in relation to its own status, that of its agents and the environment. Areas of emergence within the system's dynamics could be made visible and be addressed more easily. Another advantage over the Cournot approach would be the possibility to position the findings of such analysis in geography, space and time, something that is critical when assessing the ATS. This implies that agents are subject to change themselves, i.e., that they evolve and may disappear as well. Short of developing a full model, following an agent-based logic in a network context such as China's ATS is reckoned to be powerful enough to seriously qualify several of 
the key findings made through Cournot and to provide relevant tools for policy makers when analysing the real ATS of China.

\subsection{Heuristics}

Our approach is inductive in the sense that empirical observations should help us to recognise patterns within different elements of the Chinese ATS. The choice of where to look for such patterns and regularities (or irregularities, such as thresholds in patterns between groups of cases) needs to be guided, though. We have opted for a combined network and agent-based perspective mainly for the following reasons: first, years of applied research in the field of aviation, industrial economics and strategy have formed the author's opinion that this particular way of analysis was best suited for describing ATS. Secondly, both network economics and agent-based modelling can no longer be considered marginal disciplines that yet need to emerge more fully in scientific research. In fact, real world applications of both network and agent-based heuristics do already abound in the Aviation industry, while science still struggles to provide meaningful tools for policy making. The repeated application of general equilibrium models to problems of network structure, capacity allocation on routes, and highly stylised competition are examples of this struggle.

The question of scientific falsification of such research is legitimate, with the author reckoning that ideally this process would require a finished ABM-model against which real world developments could be simulated. Any discrepancies with real-world data would need to feed-back into model modifications. By analogy, the author sees his projections for the future development of China's ATS - which are presented in the last chapter - as equally subjected to reality-checks from the real world.

\subsection{Data}

Data for most of the statistics comes from OAGs databases. Origin-destination (OD) data provides detailed information on carriers' operations inside the People's Republic of China (PRC) and was taken from OAGs monthly reports of scheduled flights. This OD data was sampled for the months of January 2006 and January 2011, and it included traffic for Hong Kong and Macau. The data included domestic flights performed by domestic carriers only.

After compiling the data into matrices of directed city-pairs for all operating carriers, some 4,800 (2006) plus 8,400 (2011) cases were listed for the respective months of January. These filtered cases were still in a sufficiently extensive format to allow for further selection and synthesis, depending on the reference of agent for which one had to perform the analysis.

\section{Why there are HS in China}

Zhang (2010, p.189) - erroneously, as will be shown - states that the major Chinese carriers (Air China, China Eastern, China Southern) had been mainly operating on FC, rather than HS. A succinct review of the literature on HSs is presented (p.191) which mainly emphasises competitive advantages that may be obtained through such networks. To make his point, Zhang (p.190) presents graph-theoretic depictions of city-pair links 
across China for each one the three main carriers. These links emphasise connectivity, but remain un-weighted with regards to actual traffic flows, frequency, etc. This depiction is surprising as the variable for 'Frequency' of flight operations between airports were central for solving all of the Cournot model's optima in the same paper.

\subsection{Frequency distributions among China's largest airline networks}

Huber (2010, p.181) plotted rank-ordered traffic distributions (frequencies) across domestic airports for China's ten largest carriers (including the three previously mentioned). This data had covered the month of January 2007 and was plotted on a log-scale for frequency and a linear scale for each airport. Visual inspection of the ten airlines showed steeply decreasing traffic distributions (even on a log-scale) with the respective networks top-airport clearly standing over the rest in most cases. If HS can be defined as a concentration of air traffic in space and time (not shown on the chart), which per se is not incompatible with the application of Cournot, then China's top airlines even individually - can be graphically depicted by ranked-order frequency distributions. Traffic concentration in space and time by a single (or few) airlines at the same airport is to be considered essential for hub operations. In the case of an absent dominant carrier at the airport, hub advantages (density, see Brueckner and Spiller) would need to be shared among more airlines which in turn would increase transaction costs, such as for coordination, etc. Figure 1 shows such an updated ranked-order distribution for the month of January in 2011.

Figure 1 Domestic with international frequency distributions (2011)

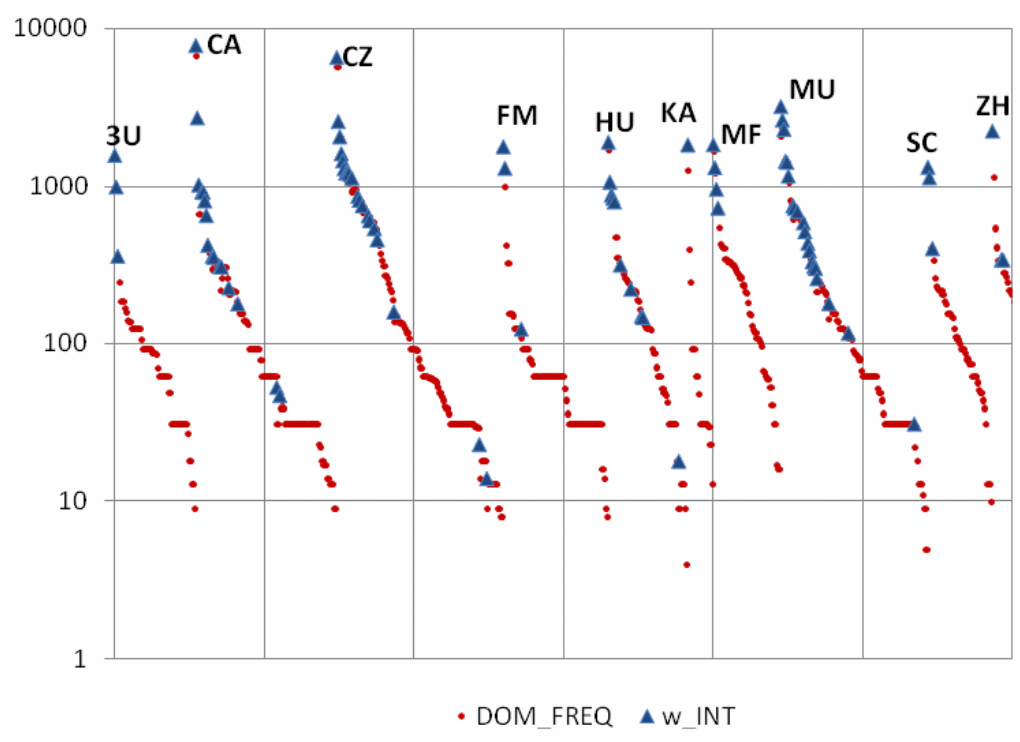

All frequencies of airlines (depicted are China's top-ten carriers, the top-thre being coded as $\mathrm{CA}, \mathrm{CZ}, \mathrm{MU})$ at airports served are ordered by decreasing rank, with red dots representing domestic traffic. For illustrative purposes, international frequency 
(cumulative to domestic) as operated by the airline at the same airport was added to the figure. It is represented by blue triangles.

As Huber (2009, p.382) had pointed out, the aggregate ranked-order distribution of all traffic within the ATS could plausibly be represented by either a power law or by exponential decay, depending on whether airlines concentrated their hub traffic on very few or more airports. In that sense, traffic concentration of China's top airlines at some hub airports may be causal for local congestion and high marginal costs related to capacity expansion for the system as a whole.

\subsection{Spatial allocation of capacity at China's airport hubs}

Figure 1 allows us selecting those airports that were the most densely operated by China's top-ten airlines. As additional criteria, we required that these airports were ranked at the least as second within the airline's network, that they provided international connectivity and that their domestic movements exceeded 1,000 during January 2011. Airports that are lower ranked within China's top-ten carriers or that may have high passenger numbers due to a high number of airlines with high density traffic may not qualify for these criteria of hub selection. By definition, we shall consider hub operations as inseparable from the presence of a dominant airline which is concentrating traffic for reasons of optimisation and competition.

Table 1 lists the ten airports that were thus identified as 'hubs'. When comparing with China's largest airports (Wikipedia, 2012a), we found significant, yet not complete, overlap: including Hong Kong, which was missing from the list, the identified hubs corresponded to eight out of the 11 largest airports in China. Only Jinan Airport, which was Shandong Airlines' (SC) main hub, ranked significantly below at rank 22. Airports that were considered among China's busiest but were not defined as a hub were Xi'an Xianyang (rank 8) and Hangzhou (rank 10). Airports that were identified as hubs for CA, $\mathrm{CZ}$ or MU were: PEK, PVG, CAN, CTU and CKG. Hubs for the remainder of China's top-ten airlines were: $\mathrm{HKG}$, SHA, SZX, KMG, XMN, and TNA.

Table 1 China's hub airports with top-three carriers' market shares (passengers, frequency, dom. city-pairs, January 2006-2011)

\begin{tabular}{llccccc}
\hline $\begin{array}{l}\text { HUB/ } \\
\text { AL }\end{array}$ & AP_NAME (\# airlines) & PAX_2011 & FRE_Jan'11 & Chg.'06 & CP_'11 & FRE/CP \\
\hline PEK & Beijing capital (17) & 78.674 .513 & 14.656 & $45.8 \%$ & 103 & 65 \\
\hline CA & Air China & & $45.6 \%$ & $10.4 \%$ & 75 & 89 \\
CZ & China Southern & & $16.9 \%$ & $-12.7 \%$ & 39 & 63 \\
HU & Hainan Airlines & & $11.6 \%$ & $-25.9 \%$ & 34 & 50 \\
\hline$H K G$ & H'Kong Int. Nat'l (12) & 53.314 .213 & 3.198 & $13.3 \%$ & 39 & 42 \\
\hline KA & Dragonair & & $39.3 \%$ & $18.9 \%$ & 16 & 79 \\
MU & China Eastern & & $19.0 \%$ & $-14.7 \%$ & 17 & 36 \\
CZ & China Southern & & $9.6 \%$ & $-52.7 \%$ & 5 & 61 \\
\hline$C A N$ & Guangzhou (15) & 45.040 .340 & 10.806 & $48.1 \%$ & 79 & 50 \\
\hline CZ & China Southern & & $52.5 \%$ & $-9.3 \%$ & 65 & 87 \\
MU & China Eastern & & $10.6 \%$ & $13.5 \%$ & 22 & 52 \\
CA & Air China & $9.0 \%$ & $-18.8 \%$ & 14 & 70 \\
\hline
\end{tabular}


Table 1 China's hub airports with top-three carriers' market shares (passengers, frequency, dom. city-pairs, January 2006-2011) (continued)

\begin{tabular}{|c|c|c|c|c|c|c|}
\hline $\begin{array}{l}H U B / \\
A L\end{array}$ & $A P \_N A M E$ (\# airlines) & $P A X \_2011$ & FRE_Jan'11 & Chg. '06 & $C P_{-}^{\prime} 11$ & $F R E / C P$ \\
\hline$P V G$ & Shang. Pudong (17) & 41.447 .730 & 6.916 & $59.2 \%$ & 62 & 64 \\
\hline MU & China Eastern & & $29.7 \%$ & $-4.0 \%$ & 37 & 56 \\
\hline $\mathrm{CZ}$ & China Southern & & $15.8 \%$ & $-24.4 \%$ & 23 & 48 \\
\hline FM & Shanghai Airlines & & $14.4 \%$ & $-25.8 \%$ & 28 & 36 \\
\hline$S H A$ & Shanghai H'qiao (15) & 33.112 .442 & 7.217 & $24.5 \%$ & 67 & 48 \\
\hline MU & China Eastern & & $30.4 \%$ & $-21.6 \%$ & 43 & 51 \\
\hline FM & Shanghai Airlines & & $23.6 \%$ & $0.1 \%$ & 37 & 46 \\
\hline $\mathrm{CZ}$ & China Southern & & $8.8 \%$ & $-25.5 \%$ & 7 & 90 \\
\hline$C T U$ & Chengdu (19) & 29.073 .719 & 7.558 & $68.7 \%$ & 69 & 38 \\
\hline $\mathrm{CA}$ & Air China & & $35.0 \%$ & $-11.2 \%$ & 48 & 55 \\
\hline $3 \mathrm{U}$ & Sichuan Airlines & & $20.4 \%$ & $-15.6 \%$ & 40 & 39 \\
\hline $\mathrm{CZ}$ & China Southern & & $11.7 \%$ & $4.2 \%$ & 14 & 63 \\
\hline$S Z X$ & Shenzhen (12) & 28.245 .738 & 7.279 & $32.4 \%$ & 57 & 51 \\
\hline $\mathrm{ZH}$ & Shenzhen Airlines & & $29.9 \%$ & $-4.6 \%$ & 40 & 54 \\
\hline $\mathrm{CZ}$ & China Southern & & $27.9 \%$ & $-1.7 \%$ & 39 & 52 \\
\hline $\mathrm{CA}$ & Air China & & $10.9 \%$ & $7.2 \%$ & 9 & 88 \\
\hline$K M G$ & Kunming (18) & 22.270 .130 & 5.843 & $51.2 \%$ & 52 & 36 \\
\hline MU & China Eastern & & $40.2 \%$ & $1.2 \%$ & 39 & 60 \\
\hline $\mathrm{CZ}$ & China Southern & & $16.4 \%$ & $-31.0 \%$ & 21 & 46 \\
\hline $\mathrm{CA}$ & Air China & & $13.8 \%$ & $-1.5 \%$ & 20 & 40 \\
\hline$C K G$ & Chongqing (19) & 19.052 .706 & 5.230 & $95.0 \%$ & 55 & 35 \\
\hline$\overline{\mathrm{CA}}$ & Air China & & $17.2 \%$ & $-25.2 \%$ & 15 & 60 \\
\hline $3 \mathrm{U}$ & Sichuan Airlines & & $18.7 \%$ & $-7.5 \%$ & 29 & 34 \\
\hline $\mathrm{CZ}$ & China Southern & & $12.8 \%$ & $-34.4 \%$ & 16 & 42 \\
\hline$X M N$ & Xiamen (16) & 15.757 .049 & 4.136 & $63.9 \%$ & 43 & 40 \\
\hline $\mathrm{MF}$ & Xiamen Airlines & & $40.3 \%$ & $-11.9 \%$ & 32 & 52 \\
\hline $\mathrm{CZ}$ & China Southern & & $12.3 \%$ & $-37.6 \%$ & 12 & 42 \\
\hline $\mathrm{CA}$ & Air China & & $11.7 \%$ & $19.1 \%$ & 11 & 44 \\
\hline$T N A$ & Jinan (13) & 7.879 .707 & 2.844 & $97.9 \%$ & 32 & 33 \\
\hline$\overline{\mathrm{SC}}$ & Shandong Airlines & & $45.1 \%$ & $-14.9 \%$ & 28 & 46 \\
\hline $\mathrm{CZ}$ & China Southern & & $11.8 \%$ & $-27.8 \%$ & 11 & 31 \\
\hline MU & China Eastern & & $11.1 \%$ & $-19.8 \%$ & 8 & 40 \\
\hline
\end{tabular}

Table 1 shows that China's top-three airlines indeed held significant - on occasions dominant - control over China's most important airports. In all instances, at least two out of the three highest market-shares of airlines at airport hubs came from this group of airlines (CA, CZ, and MU). This level of interconnectedness among China's hub airports clearly stresses the role of said three airlines for China's ATS as a whole. It also raises questions about the agents' quality of interaction - or degree of independency - in route planning regarding the remaining major airline networks. 


\subsection{A qualification of the graph-theoretic perspective}

Zhang (2010, p.190) suggested a FC network structure for China's three main carriers (CA, MU, and CZ) based on a graph-theoretic depiction of city-pair links. The graphs below, in contrast, take into account only the densest (highest frequency) routes between all airports with the scale of frequency represented through the thickness of the link. The colours of the links were attributed to the individual airline that showed the highest market-share for the particular route. There were 27 (15) Chinese airlines accounted for in 2011 (2006), all of them being grouped into one of four airline groups (see Appendix for full list of airline groups, including some network characteristics): ${ }^{2}$ (red) comprised the three leading carriers CA, MU, CZ; (green) represented the other seven main operators that were also shown in Figure 1; (blue) stood for smaller to medium sized airlines, most of which had entered service after 2006; and (grey) defined regional carriers, i.e., a classification made by the prevalent aircraft technology of the airline. Putting weights on the links clearly shows a preference for interconnecting the biggest airports through highest frequency traffic. In that sense, the logic of 'preferential attachment' (Albert and Barabási, p.47f) would extend to frequency, not only to connectivity w.r.t. city-pairs. The graph-theoretic layout was obtained by using UCINETs algorithm for geodesic distances, including the node repulsion and equal edge length function (Borgatti et al., p.40f).

Figure 2 Only maximum frequencies between airports (January 2006)
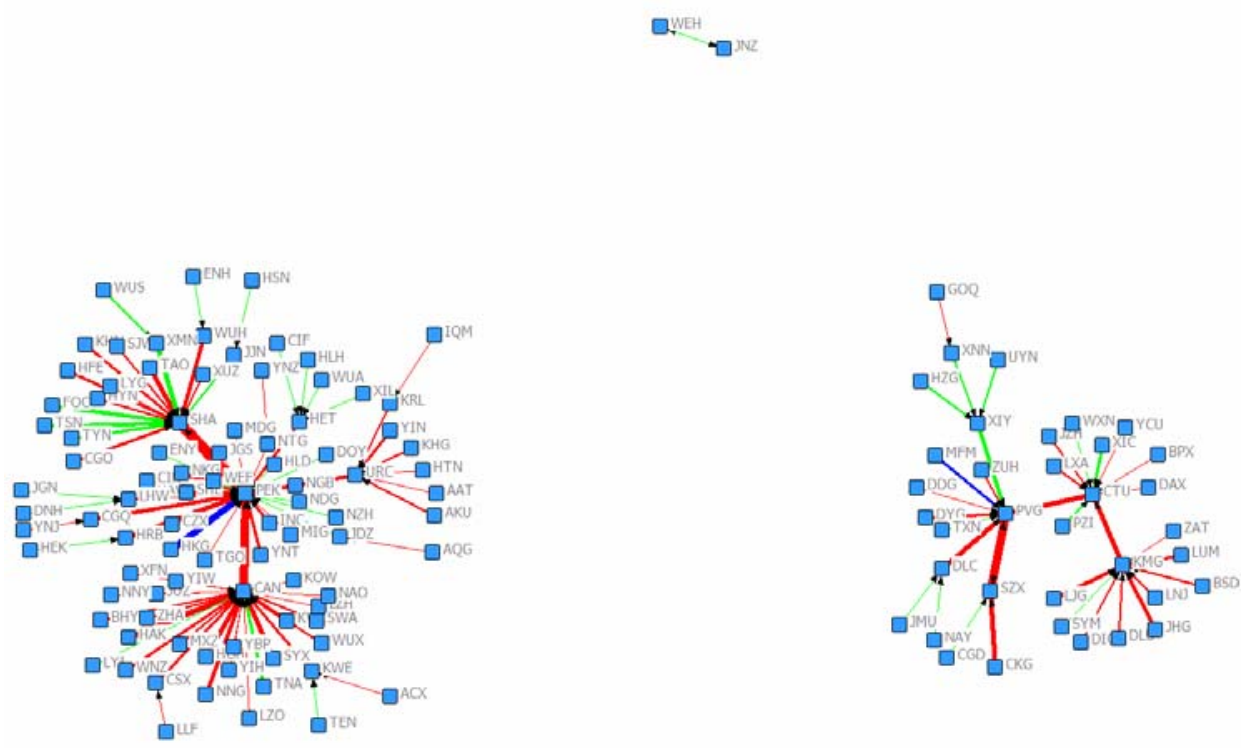

Figure 2 shows how airports' maximum frequencies were feeding into relatively few major airports, which in turn formed two separate complexes (one around SHA-PEK-CAN, the other around PVG-CTU-KMG). Since both PVG and SHA were in the same city, the complex could geographically be considered as one. More importantly, highest frequency connections within the Chinese ATS appeared to be dominated by the three main carriers (red links), with the remaining seven major carriers (green) mainly 
feeding into both airports in Shanghai. A few selected routes (green) fed into three to four airports that had not been identified as hubs before (see Figure 1) and which also appear as spoke airports within the graph. Medium traffic frequency between HKG and PEK was dominated by a smaller airline (blue link).

Figure 3 Only maximum frequencies between airports (January 2011)

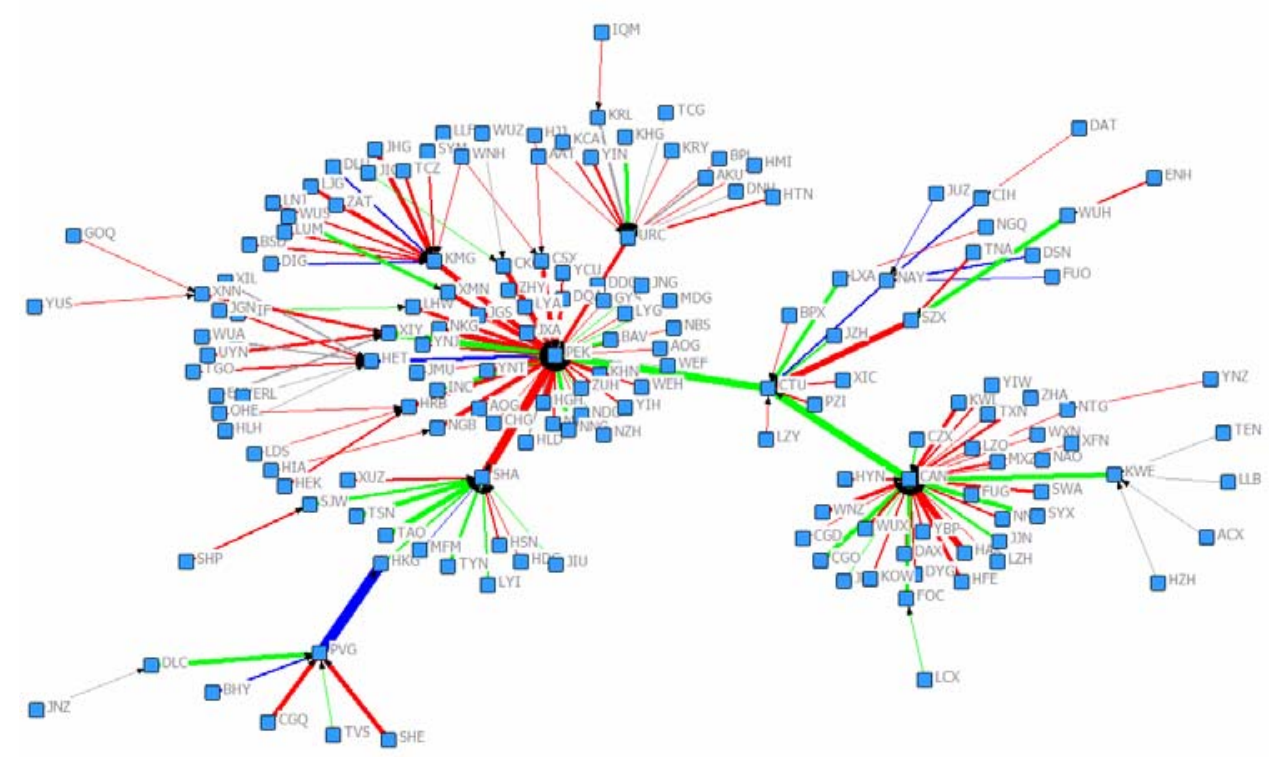

By 2011 (Figure 3), the overall system appeared both more extensive (grown in scope and size) as well as being more integrated. Identified hub airports were strongly interconnected and committed their maximum frequencies to each other respectively. China's major airlines (red plus green) still held the highest shares for capacity among almost all of the airports' maximum frequency links, with the green part taking over an increasing stake. HKGs maximum traffic has increased and shifted from PEK (in 2006) to PVG (blue link). The network organisation of the entire system appeared more hierarchical, with more spoke airports themselves serving as feeders for maximum frequencies from yet smaller airports. In many instances, these spoke-to-spoke links were controlled by 'red' CA, CZ or MU - significantly more than there were such cases for 'green' carriers. Some regional airlines (grey links) allowed for thinner links (connectivity) from a few sub-spoke airports.

\section{Decomposing the HS network}

When comparing the un-weighted, FC approach of Zhang with the (grouped) agent-based network graphs, an elaborate HS system becomes visible. This network status, however, may not present a social optimum as marginal costs for airport expansion at hubs are likely to exceed marginal returns, mostly to the detriment of passengers, due to congestion costs with regards to connecting flights, etc. Selective de-peaking at hub airports or even imposing a FC structure, however, would require China's top ten airlines 
to abandon their hub strategies and to review their mutual concentration of traffic frequency at these hubs. Not only is this unlikely to happen, but severe disruption to the entire system would be a most plausible consequence. Given the degree of dependency of spoke airports and even spoke-spoke connections on these largest operators, sustained growth and connectivity at smaller and even medium-sized airports would be at risk. This prediction is consistent with Huber (2010, p.183) who sees excessive concentration of traffic by the top-ten carriers at hubs which induces negative variance in wider parts of the network. As a result, because of excess frequencies being funnelled from other hubs as well as spokes into the biggest airlines' hubs, the remaining airports remain compromised in serving their local 'potential' demand. In other words, measures for de-peaking or re-allocation of flight schedules and their frequencies towards a less concentrated and more FC ATS require a distinction of types of linkages between the various airports.

\subsection{Distinguishing types of airports}

A graph-theoretic network can be decomposed into the constituent airports, while attributing different roles to them. A heuristic that was explained above is the identification of hub airports. Due to findings in literature (see Zhang), the role of CA, $\mathrm{CZ}, \mathrm{MU}$ is most fundamental and therefore - by extension - their respective hubs (Hub_1) shall be considered apart from those of the remaining seven major airlines (Hub_2). Airports that are directly connected to these hubs are treated as 'Spoke' airports. A distinction between spokes connecting to Hub_1 from those connecting to Hub_2 shall not be made at this point, mainly because a great number would be connected to both types of hubs - a finding that may be better analysed in the following section. The class of 'Sub-Spoke' refers to airports that are not linked to hubs, but either to spoke airports or to each other.

Table 2 Network decomposition (all frequencies) into airport classes

\begin{tabular}{lcccccccc}
\hline \multirow{2}{*}{ Hub_1 } & & AP & OD & $a_{-} O D$ & $F R E$ & $a_{-} F R E$ & FRE/OD & $m s_{-} T 3$ \\
\hline \multirow{2}{*}{ Hub_2 } & 5 & 365 & 73 & 45.779 & 9.156 & 125 & $63.0 \%$ \\
& Chg.'06 & $0.0 \%$ & $36.2 \%$ & $36.2 \%$ & $52.4 \%$ & $52.4 \%$ & $11.9 \%$ & $-10.0 \%$ \\
& Jan'11 & 6 & 293 & 49 & 29.904 & 4.984 & 102 & $36.8 \%$ \\
\multirow{2}{*}{ Spoke } & Chg.'06 & $0.0 \%$ & $26.8 \%$ & $26.8 \%$ & $44.0 \%$ & $44.0 \%$ & $13.6 \%$ & $-22.2 \%$ \\
& Jan'11 & 129 & 1.403 & 11 & 93.253 & 723 & 66 & $52.3 \%$ \\
\multirow{2}{*}{ Sub-spoke } & Chg.'06 & $29.0 \%$ & $50.2 \%$ & $16.4 \%$ & $90.4 \%$ & $47.6 \%$ & $26.8 \%$ & $-9.9 \%$ \\
& Jan'11 & 24 & 30 & 1 & 1.566 & 65 & 52 & $64.8 \%$ \\
\multirow{2}{*}{ Total } & Chg.'06 & $33.3 \%$ & $42.9 \%$ & $7.1 \%$ & $89.1 \%$ & $41.8 \%$ & $32.4 \%$ & $20.4 \%$ \\
& Jan'11 & 164 & 2.091 & 13 & 170.502 & 1.040 & 82 & $53.9 \%$ \\
& Chg.'06 & $27.1 \%$ & $43.8 \%$ & $13.1 \%$ & $69.5 \%$ & $33.3 \%$ & $17.8 \%$ & $-6.2 \%$ \\
\hline
\end{tabular}

These classes of airports are compared across the following variables: number of airports within a given class ('AP'), number of (directed) OD performed by all airports within a given class ('OD'), average number of OD links by each airport ('a_OD') of a given 
class, number of scheduled flight movements ('FRE') of a given airport class, average number of flights per airport within same class ('a_FRE'), density or average number of movements per directed route ('FRE/OD') and cumulative market-share ${ }^{3}$ of the prominent three airlines (CA, CZ, and MU) within the same airport class ('ms_T3'). Summary results are shown for January 2011 as well as changes over the five-year period, i.e., with January 2006 as the base.

The results present information about the Chinese domestic ATS and the contribution of each of its classes of airports: clearly, this summary decomposition reflects the completely distinct features and - by inference - roles of the various classes: Hub_1 maintained its lead over Hub_2 airports in terms of both scale and scope of operations, with each variable having grown faster than with the $\mathrm{H}_{-} 2$ class. Market-share of T3 airlines continued to be dominant at $\mathrm{H}_{-} 1$ and strong at $\mathrm{H}_{-} 2$, although this share had dropped considerably for H_2. Spoke airports were the most numerous within the system and had grown by $29 \%$ during the period. Spoke airports collectively represented some $65 \%$ of all directed links within the network and almost $55 \%$ of all movements. The average number of links (connectivity) of a 'Spoke' airport was less than $18 \%$ that of the average hub, while the average frequency of a 'Spoke' was slightly above $10 \%$ of the average hub's. The average density on a route departing from a 'Spoke' airport was 66 , or slightly above twice per day, while on a route departing from a hub it was between 3.4 (Hub_2) and 4 (Hub_1). Although all of these network variables had grown for 'Spoke' airports, growth for the average number of ODs was significantly trailing behind that of hubs: this may be explained by the fact that hubs continued to grow new routes to new airport-spokes, while 'Spokes' focused on increasing traffic density on existing routes. As for the 'sub-spokes', they were mostly connected to only one other airport and could be seen as a point-to-point, marginal and fragmented, system with less than two daily flights (average) on their OD links. This airport class of sub-spokes was upheld by top-three carriers (with a $65 \%$ market-share), possibly through public-service-obligations, or similar contracts.

\subsection{HS connected-route differentiation for types of traffic flows}

The following analytic template is not an ad-hoc invention, but represents the result of several cycles of analysis of raw OD data and synthesis of distinct levels of matrix aggregation for the Chinese domestic ATS. It is an abstraction of the Matrix results for the different original agents (airlines, airports) and the patterns (topology) found within the distinct classes of airports and airlines.

Hub airports had been identified before. Within China's ten leading airlines which were defined as eligible for hub operations, the top three airlines (CZ, MU, and $\mathrm{CA}$ ) needed to be distinguished due to their prominence and stronger influence on the system as a whole: these were associated with Hub_1, the remaining 7 airlines (HU, ZH, MF, SC, 3U, FM, and KA) operated on airports that were coded Hub_2. Routes that connected HH_1 airports on both ends were defined as HH_1, those connecting HH_2 airports on both ends were HH_2 and those hub-to-hub links that had the other class on its end were considered as HH_3. 
Figure 4 A template for analysing the Chinese ATS (see online version for colours)

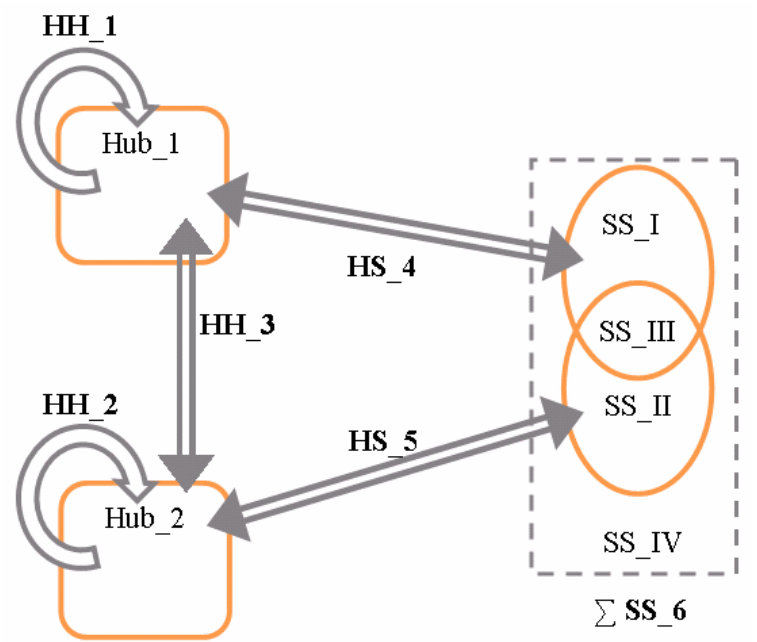

Another type was hub-spoke links, i.e., links that presented a hub on one end and a non-hub at the other. Depending on whether the hub side was part of Hub_1 or Hub_2, these hub-spoke links were either named HS_4 or HS_5, respectively. The non-hub airports were not only connected to hubs, but also to other airports, which can be named by convention - sub-spokes. The ensemble of such sub-spoke links can be summarised as $\sum$ SS_6, although there are different classes: SS_I stands for sub-spoke links that are connected to a spoke airport which in turn is connected to one or several Hub_1 airports only. SS_II is a sub-spoke link to/from an airport spoke with a connection to one or several Hub_2 airports solely. SS_III sub-spokes are linked to airports with one or several connections to both Hub_1 and Hub_2 type of airports. SS_IV represents sub-spokes without any connection to Hub airports; in a sense, they are self-organised. A final group could be classified as SS_0 (not shown) and represents inexistent sub-spoke connections for an airport that presents hub-spoke linkages. All of these linkages are additive with regards to allocated frequencies, i.e., the sum would present the total capacity (in air traffic movements) within the Chinese ATS during the observation period.

\subsection{A changing topology for China's ATS}

The following table shows empirical results after applying the template to real flight data for the month of January in 2006 versus that of 2011.

Among hub-hub routes, capacity allocation (frequency of movements) between the five biggest airports of 'red' airlines continues to be higher than airline movements between the six biggest hubs of 'green' airlines. However, growth in the latter was much greater. Frequency of flights between both kinds of hubs was higher compared to capacity within each kind, which can - in part - be explained mathematically: the possible number of connections between all hub airports being $(5+6)^{2}-11$, the potential number of $\mathrm{HH} \_3$ routes would be $\left((5+6)^{2}-11\right)-\left(5^{2}-5\right)-\left(6^{2}-6\right)=60$. This well exceeds the number of potential HH_1 plus HH_2 routes $(=50)$. 'Red' airlines (details can be obtained from the author) had allocated over $53 \%$ of their total within-hub movements to HH_3 routes in January 2006, a percentage that got closer to 50\% by 2011 . 
'Green' airlines' had some $70 \%$ of their intra-hub capacity allocated to HH 3 during January 2006, a number that was reduced to about $60 \%$ in 2011 . Clearly, 'green' continued to grow preferentially such routes to/from 'red' hubs, while the 'red' airlines maintained a more even balance between HH_1 and HH_3. This pattern suggests controlled saturation of the 'red' hubs by both 'red' and 'green'.

Figure 5 Frequencies on connected-route types and changes (2006-2011)

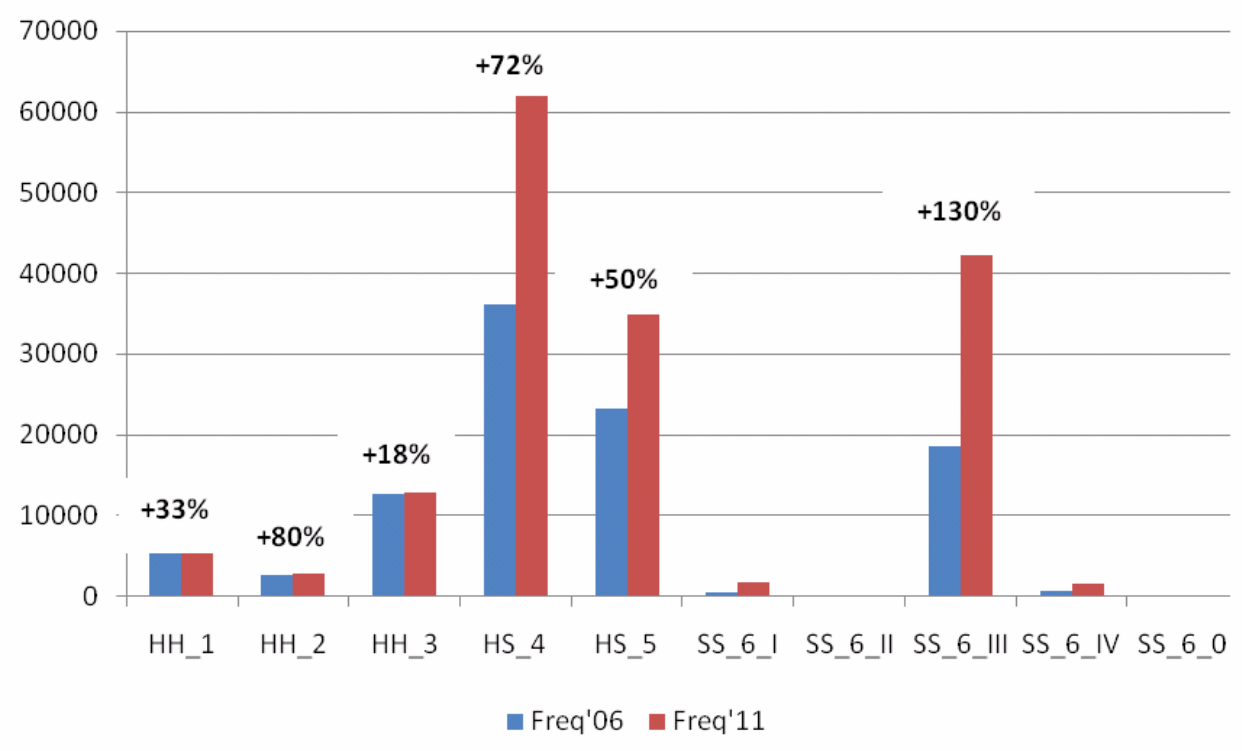

As for movements that connected hubs and spokes, a distinction was made between 'red' hubs and their spokes (HS_4) and 'green' hubs and their spokes (HS_5). Unsurprisingly, HS_4 movements had exceeded those of HS_5 in January 2006, but also continued to grow much faster during the observation period. On one side, the number of hub-spoke movements increased its feed into intra-hub movements, suggesting a continued emphasis on connecting traffic at the hubs. On the other side, the hierarchical relationship between 'red' and 'green' hubs became more emphasised as increases in HH_3 movements had not diminished the difference between HS 4 and HS_5 frequencies, quite the opposite. It shall be noted that this increase in difference of flight movements between HS 4 and HS 5 is not contradictory to a possible spatial segmentation between HS networks that are dominated by either 'red' or 'green' airlines, although they remained highly interconnected through their hubs.

Another striking finding by applying the template is the near non-existence of sub-spokes that are linked to airports with either solely HS_4 or solely HS_5 movements. The by-far dominant type of sub-spokes is linked to airports which provide links to both 'red' and 'green' hubs (SS_6_III). It is these sub-spokes that have grown to such an extent that the topology of China's overall ATS was altered considerably during the observation period. This suggests more feed into traffic at spoke airports (which in turn provide routes to both 'red' and 'green' hubs), which could be interpreted as a buffer to by-pass transfers from the very hubs and to increase traffic at the HS legs instead. It may 
be considered a preliminary, yet indispensable, step to grow these spoke airports into hubs at a later stage.

\subsection{The role of airline agents}

China's red airlines clearly dominate intra-hub traffic, including a very strong presence at the green airlines' hub airports (compare with Figure 5). The somewhat limited growth on these routes cannot be fully explained by higher costs for expansion at these hubs, as feeder routes to/from spokes continued to add much more capacity, in particular to the five hubs dominated by red carriers. Blue airlines, including numerous entrants, had almost no presence at intra-hub traffic but had built up most of their capacities on hub-spoke routes. As with those HS routes that were feeding to/from the six hubs dominated by green airlines, red airlines almost stopped adding capacity thus leaving more control within this part of the system to green airlines.

Figure 6 Connected-route types for airline groups (frequency, 2006-2006)

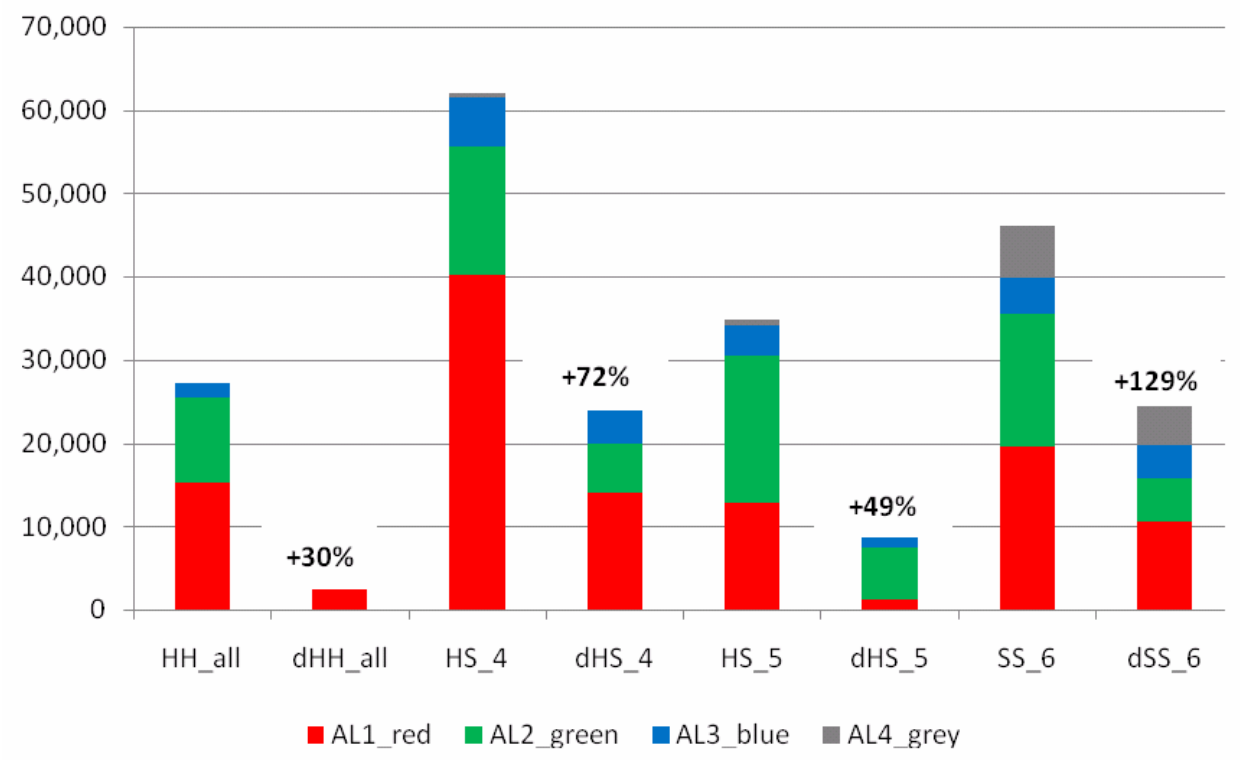

It is noteworthy that red airlines maintained (actually doubled) their presence on spoke-spoke routes, which is evidence of its ongoing deep penetration of the Chinese ATS. Green presence at spoke-spoke was not quite as important and in fact had increased only by about half of that of red agents. Most of blue capacity on these routes had not existed in 2006 and is about half of blue capacity that was deployed on hub-spoke routes. Capacity growth of green and blue agents was more biased towards hub-spoke routes while red agents maintained their strong presence in the spoke-spoke segment of the system. Regional carriers (grey agents) do not appear to play any role in feeding into hubs, but remain focused on selected spoke-spoke traffic. The most dynamic regional carrier appears to be Tianjin Airlines (GS), which for 2012 was reckoned to hold 90\% of 
China's regional traffic and operated from its own hub in Tianjin Binhai International Airport (Wikipedia, 2012b).

What can plausibly be inferred from the above is an evolutionary type of growth of China's ATS through both individual and collective behaviour of classes of agents. Although new entry (almost no exits) occurred during the observation period, any potential for competition would need to be limited to distinct parts of the network, and be subject to system-wide control by the 'red' carriers. An emerging spatial separation of hub-spoke routes into 'red' and 'green' networks need not diminish control by the former, due to dominance and dependency at the hub level. In spite of the overwhelming presence of 'red' agents in the system, it may yet not suffice to explain the system's nuanced and adaptive behaviour by these agents alone: a supreme regulatory/planning authority may still be instrumental for improving such multi-agent, multi-hub, network-wide outcomes.

\section{A system-wide architecture for balanced hub-spoke growth}

The paper shows that in spite of its mathematical elegance, the application of Cournot to the Chinese ATS may prove inadequate in the end: China's ATS as a real system is growing in a sustained manner, not by avoiding HSs, but by using it in a complex, adaptive, interacting and yet controlled manner. The system as such can be regarded as evolutionary with a high potential of growing new HS airports within the system. The agent-based perspective allows us to discuss trade-offs in allocation of frequency between different kind of agents (airports versus airlines) as within the same kind, i.e., for classes of airlines. We were able to decompose the system into new agent-types, such as route elements, and to discuss their distinct roles with regards to behaviour of the aforementioned agents. Short of presenting a quantitative and validated simulation of the future evolution of the Chinese ATS, some emerging patterns and trends can be inferred nonetheless - and contrast with the dichotomous FC versus single HS choice that seems to be advocated by Zhang.

A plausible outlook, i.e., a consistent spatial-temporal extrapolation of the system, cannot ignore the issue of de-peaking China's main hubs. Our analysis leaves no doubt that China's top-three 'red' carriers will have to determine the resolution of this issue, as the entire ATS is likely to be affected by their behaviour: if reducing feeder traffic from/to their hubs, their service on spoke-spoke routes is likely to be impacted as well. If hub-hub traffic is to be reduced, new slots might possibly become available for new entrants, which in turn may have indirect effects on the relationship among hub incumbents. Moreover, China's 'red' airlines will be very careful about keeping their control on the system and being able to shape the structure of China's domestic ATS also in the future. This is why frequency on hub-hub routes may more easily get reduced by 'green' airlines or by 'red' and 'green' in a proportional manner. A biased reduction of 'red' frequency would risk a shift in power towards 'green' carriers, which seems unrealistic in the Chinese context.

One option for maintaining structural balance between 'red' and 'green' at saturated hub airports would be to allow for entry by 'blue' carriers at the hub-spoke routes, even allowing for dense frequencies. Such a development has already started during the observation period and it would facilitate the 'blue' carriers' growth and organisation of traffic around smaller hubs of their own. Very dense connectivity that is being maintained 
through the larger 'red' and 'green' airlines on these routes could thus be reduced to significantly lesser levels. With these 'blue' airlines growing connectivity and frequency around their own hubs, they could progressively take over control on these hub-spoke routes, requiring lower frequencies at the earlier defined hubs. Such spatial segmentation of control within the Chinese ATS is already under way at a higher level: the 'green' airlines have emphasised growing frequency on hub-spoke routes to/from hubs that they already dominate, mostly to the local detriment of 'red' carriers. In the end, this made capacity growth on 'green' spoke-spoke routes viable (although spoke-airports involved were also linked to 'red' hubs at the same time). The sustained entries of new 'blue' and 'grey' airlines may follow the same logic of spatial concentration (hubbing) at a smaller scale, a long run scenario that yet needs to emerge more fully.

With the (medium-term) emergence of smaller and medium-sized hubs, the sources for marginal revenues through connecting passengers would necessarily become more spatially dispersed: on the one side, airport charges at these airports cannot afford to be as high as with big hubs (otherwise they would lose customers to other connecting airports), on the other side these marginal revenues are more likely to be invested into capacity expansion from much smaller scales. Insofar, the spatial dispersion of 'external' marginal revenues is deemed to both increase system-wide capacity in a more balanced way as well as to foster (bounded) competition between these smaller hubs. An emerging multi-agent, multi-hub system - being formed through spatial concentration of bounded local capacity by multiple airlines - may collectively create a positive competitive balance against the larger hubs. This is why control of certain routes, i.e., hub-spoke routes to 'red' or 'green' hubs, should gradually be left to 'blue' or 'grey' airlines in the longer run, provided that they are able to grow their own networks.

A final observation is that China's top three airlines were able to control the network system, while allowing for entry and growth around certain airports. In the same time, these 'red' carriers have maintained a very deep presence throughout the ATS, including at smaller airports and on spoke-spoke routes. One could plausibly interpret the role of China's 'red' agents as evolutionary-catalytic, i.e., as one of opening routes, establishing connectivity among a diverse set of airports, and even encouraging competing connections to 'green' hubs. Its spatial presence seems adaptive and was shown to be able to forgo local dominance on critical occasions. There is no indication of rigid planning in the sense that China's 'red' (and probably 'green' agents at a later stage) were unable to adjust their network strategies over time.

\section{References}

Albert, R. and Barabási, A.L. (2002) 'Statistical mechanics of complex networks', Reviews of Modern Physics, January, Vol. 74, pp.47-97.

Borgatti, S.P., Everett, M.G. and Freeman, L.C. (2002) UCINET 6 for Windows Software for Social Network Analysis - User's Guide, Analytic Technologies, Inc., Lexington.

Brueckner, J.K. and Spiller, P.T. (1994) 'Economies of traffic density in the deregulated airline industry', Journal of Law and Economics, Vol. 37, No. 2, pp.379-415.

Huber, H. (2009) 'Statistical mechanics for analytic planning: an application to domestic air traffic in China', International Journal of Management \& Network Economics, Vol. 1, No. 4, pp.378-393.

Huber, H. (2010) 'Planning for balanced growth in Chinese air traffic: a case for statistical mechanics', Journal of Air Transport Management, Vol. 16, No. 4, pp.178-184. 
Railsback, S.F. and Grimm, V. (2011) Agent-Based and Individual-Based Modeling: A Practical Introduction, Princeton University Press, Princeton.

Wei, W. and Hansen, M. (2006) 'An aggregate demand model for air passenger traffic in the hub-and-spoke network', Transportation Research Part A, Vol. 40, No. 6, pp.841-851.

Wikipedia (2012a) List of the Busiest Airports in the People's Republic of China, CAAC [online] http://en.wikipedia.org/wiki/China\%27s_busiest_airports_by_passenger_traffic (accessed 28 May 2012).

Wikipedia (2012b) Tianjin Airlines [online] http://en.wikipedia.org/wiki/Tianjin_Airlines (accessed 29 May 2012).

Zhang, Y. (2010) 'Network structure and capacity requirement: the case of China', Transportation Research Part E, Vol. 46, No. 2, pp.189-197.

\section{Notes}

1 This may largely be due to his assertion that the Chinese ATS was already driven by point-to-point rather than HS.

2 A description of the criteria that allowed for the airline grouping can be obtained from the author by request.

3 Market share was determined as a fraction of carriers' movements. 


\section{Appendix}

Table A1 Airline networks: scale, frequency and scope

\begin{tabular}{|c|c|c|c|c|c|c|c|c|}
\hline Carrier & Code & Size_AP & No_OD & $M x_{-} F R E$ & $F R E \_A P$ & $M x \_S E A T$ & $I N T \_A P$ & $\begin{array}{l}\text { Table } \\
2006\end{array}$ \\
\hline China Southern & $\mathrm{CZ}$ & 111 & 823 & 5,671 & 345 & 151 & 20 & Yes \\
\hline China Eastern & MU & 98 & 635 & 2,346 & 268 & 130 & 20 & Yes \\
\hline Air China & $\mathrm{CA}$ & 94 & 403 & 6,687 & 248 & 157 & 16 & Yes \\
\hline Group 1 (red) & Avg.: & 101 & 620 & 4,901 & 287 & 146 & 19 & \\
\hline Hainan Airlines & $\mathrm{HU}$ & 53 & 314 & 1,694 & 218 & 163 & 10 & Yes \\
\hline Shenzen Airlines & $\mathrm{ZH}$ & 54 & 241 & 2,174 & 193 & 152 & 4 & Yes \\
\hline Xiamen Airlines & $\mathrm{MF}$ & 45 & 299 & 1,668 & 277 & 126 & 4 & Yes \\
\hline Shandong Airlines & $\mathrm{SC}$ & 43 & 202 & 1,284 & 178 & 144 & 3 & Yes \\
\hline Sichuan Airlines & $3 U$ & 54 & 208 & 1,545 & 130 & 143 & 3 & Yes \\
\hline Shanghai Airlines & FM & 70 & 202 & 1,701 & 106 & 131 & 3 & Yes \\
\hline Dragonair & KA & 17 & 32 & 1,258 & 148 & 193 & 1 & Yes \\
\hline Group 2 (green) & Avg.: & 48 & 214 & 1,618 & 178 & 150 & 4 & \\
\hline Hong Kong Airlines & $\mathrm{HX}$ & 8 & 14 & 229 & 57 & 202 & 1 & No \\
\hline Hong Kong Express & UO & 6 & 10 & 158 & 53 & 164 & 1 & Group 4 \\
\hline Air Macau & NX & 11 & 20 & 311 & 57 & 142 & 1 & Yes \\
\hline Spring Airlines & $9 \mathrm{C}$ & 34 & 73 & 496 & 64 & 180 & 0 & No \\
\hline Grand China Air & $\mathrm{CN}$ & 6 & 10 & 186 & 62 & 180 & 0 & No \\
\hline China United & $\mathrm{KN}$ & 25 & 48 & 832 & 70 & 171 & 0 & No \\
\hline Juneyao Airlines & $\mathrm{HO}$ & 34 & 73 & 620 & 67 & 155 & 0 & No \\
\hline China West & PN & 23 & 48 & 465 & 54 & 141 & 0 & No \\
\hline Beijing Capital & JD & 27 & 80 & 263 & 86 & 140 & 0 & No \\
\hline Chengdu Airlines & EU & 25 & 62 & 430 & 61 & 124 & 0 & Yes \\
\hline Lucky Air Co. & $8 \mathrm{~L}$ & 31 & 68 & 806 & 79 & 122 & 0 & No \\
\hline Hebei Airlines & NS & 6 & 10 & 155 & 52 & 110 & 0 & No \\
\hline Cathay Pacific & $\mathrm{CX}$ & 3 & 4 & 74 & 50 & 294 & 1 & 2006 \\
\hline Group 3 (blue) & Avg.: & 18 & 40 & 387 & 62 & 163 & 0 & \\
\hline TianJin Airlines & GS & 52 & 160 & 666 & 100 & 83 & 1 & No \\
\hline China Express & G5 & 20 & 40 & 181 & 45 & 50 & 0 & No \\
\hline Okay Airways & $\mathrm{BK}$ & 13 & 28 & 186 & 76 & 50 & 0 & Group 3 \\
\hline Joy Air & JR & 6 & 10 & 142 & 68 & 50 & 0 & No \\
\hline Group 4 (grey) & Avg.: & 23 & 60 & 294 & 72 & 58 & 0 & \\
\hline
\end{tabular}

